\title{
Detección de Borrelia burgdorferi sensu lato en perros y sus garrapatas en comunidades rurales de Yucatán, México
}

\author{
Analilia Solís-Hernández ${ }^{1}$, Roger Iván Rodríguez-Vivas ${ }^{1 *}$, María Dolores Esteve-Gasent ${ }^{2}$ \\ \& Sandra Luz Villegas-Pérez ${ }^{1}$ \\ 1. Laboratorio de Parasitología, Campus de Ciencias Biológicas y Agropecuarias. Facultad de Medicina Veterinaria y \\ Zootecnia. Universidad Autónoma de Yucatán. Km 15.5 carretera Mérida-Xmatkuil. CP. 97000. Mérida, Yucatán, \\ México; analilia.solis@gmail.com,rvivas@correo.uady.mx, sandra.villegas@correo.uady.mx \\ 2. Department of Veterinary Pathobiology, College of Veterinary Medicine and Biomedical Sciences, Texas A\&M \\ University, College Station, TX-77843, USA; MEsteve-Gassent@cvm.tamu.edu \\ * Correspondencia.
}

\section{Recibido 02-VI-2017. Corregido 18-VIII-2017. Aceptado 20-IX-2017.}

\begin{abstract}
Detection of Borrelia burgdorferi sensu lato in dogs and its ticks in rural communities of Yucatán, Mexico. In Mexico, the distribution and the presence of pathogenic genospecies of B. burgdorferi in dogs and their ticks has not been extensively investigated. The study of canine borreliosis is acquiring greater relevance, since the dog is considered to be an important sentinel for pathogens pertaining to the complex Borrelia burgdorferi sensu lato; in addition, dogs could be playing a key role in the spread of ticks from forested areas into the domestic environment. This study aimed to detect and estimate the prevalence of B. burgdorferi s.l. in dogs and their ticks in two rural communities of Yucatán, Mexico. In each community, 50 houses were visited, where 144 blood samples from dogs were studied by puncture of the saphenous vein, as well as the collection of their ticks. To detect the presence of $B$. burgdorferi s.l. in these samples, the conserved gene flaB, $p 66$ and $\operatorname{sspC}$ were PCR amplified. A total of 144 dog blood samples, and 846 of ticks were obtained from the examined animals. Considering tick species, Rhipicephalus sanguineus sensu lato (786 / 846) was common, while Ixodes affinis (33 / 846), and Amblyomma mixtum (27 / 846) resulted less frequent. As per gene conservation, the prevalence of B. burgdorferi in canine blood was $17.3 \%(25 / 144)$ to flaB, $12.50 \%(18 / 144)$ for $p 66$ and $1.38 \%(2 / 144)$ for the $\operatorname{sp} C$ gene. Within the analyzed ticks, $R$. sanguineus s.l. had a prevalence of $0.89 \%$, A. mixtum $5.88 \%$ and $I$. affinis $15.15 \%$, being this last species the one that presented higher prevalence. Two dogs and their ticks I. affinis were positive to the flaB gene. Only a tick R. sanguineus s.l. was positive to the gene $p 66$ and no tick species was positive the $\operatorname{sp} C$ gene. This study confirmed the existence of $B$. burgdorferi s.l. in dogs and their ticks in rural communities of Yucatán, Mexico. The detection of Borrelia in dogs may be an important criterion for the evaluation of the risk of borreliosis in humans, since the dog can be used as an epidemiological indicator for the identification of new outbreaks of this disease. Rev. Biol. Trop. 66(1): 428-437. Epub 2018 March 01.
\end{abstract}

Key words: Borrelia burgdorferi s.l., Lyme disease, dogs, Ixodes affinis, Rhipicephalus sanguineus s.l., Amblyomma spp., Yucatán, Mexico.

La enfermedad de Lyme (EL) es una enfermedad zoonótica producida por espiroquetas del complejo Borrelia burgdorferi sensu lato (s.l.) que afecta al humano y a los animales (Glenny, Mendoza, \& Falconi, 2004). Es transmitida por garrapatas de la familia Ixodidae (Burgdorfer, Hayes, \& Corwin, 1989), siendo Ixodes ricinus el vector más común en la transmisión de la infección en Europa e
I. scapularis e I. pacificus en Estados Unidos (E.U.) (Mannelli, Bertolotti, Gern, \& Gray, 2012). Hasta el momento, el Centro de Control y Prevención de Enfermedades de los E.U. (CDC, por sus siglas en inglés) sólo reconoce a la EL en E.U. y Euroasia, pero no en Centro y Sudamérica (CDC, 2015). La importancia de la EL en México queda por esclarecerse, a pesar de los distintos estudios que se han realizado 
sobre la distribución de la garrapata Ixodes, no sólo en los estados del noreste mexicano incluyendo la zona transfronteriza de Texas y México (Feria et al., 2014), así como a lo largo del centro y sureste del país, incluyendo la península de Yucatán (Solís-Hernández, Rodríguez-Vivas, Barrera-Pérez, Esteve- Gassent, \& Apanaskevich, 2015; Rodríguez-Vivas et al., 2016). En México, desde 1991 se reportaron los primeros casos sugestivos a EL en pacientes de Sinaloa y Monterrey (Arroyave \& Támez, 1994). Posteriormente, en 2007 se reportaron los primeros casos de EL autóctonos en humanos procedentes del Valle de México y Quintana Roo (Gordillo-Pérez et al., 2007).

Entre los hospederos domésticos de Borrelia burgdorferi s.l., el perro es el más estudiado ya que actúa como hospedero de Ixodes spp. y otras especies de garrapatas (Smith et al., 1993). El perro es considerado hospedero incidental que adquiere la infección durante la alimentación de las formas maduras de las garrapatas infectadas, mientras que las formas inmaduras tienen una mayor preferencia alimentaria por pequeños mamíferos (especialmente roedores), aves o reptiles (Krupka \& Straubinger, 2010). Algunos autores mencionan que aunque no hay evidencia de que la EL del perro suponga un riesgo para la salud pública, estos son considerados los reservorios más importantes de garrapatas en el ambiente doméstico y pueden ser fuente intermediaria para la infestación de garrapatas en el humano (Levy \& Magnarelli, 1992; Straubinger, 2000).

En México, los estudios sobre la frecuencia y distribución de B. burgdorferi s.l. en perros son escasos. En la zona norte y centro del país (Monterrey, Nuevo León y Distrito Federal) se observó una seroprevalencia del $16 \%$ (136 / 850), y en un estudio preliminar realizado en el 2003 en Mexicali, Baja California, se reportó una prevalencia de $7.4 \%$ (7 / 94) en perros infestados por la garrapata Rhipicephalus sanguineus (Salinas-Meléndez, Tamez-González, Welsh-Lozano, \& BarreraSaldaãna, 1995; Salinas-Meléndez, ÁvalosRamírez, Riojas-Valdez, \& Martínez-Muñoz, 1999; Tinoco-Gracia et al., 2008).
En México se ha reportado que el perro es hospedero de varias especies de garrapatas, que incluyen Rhipicephalus sanguineus sensu lato, Dermacentor nitens, Amblyomma mixtum, A. sabanerae, A. parvum, A. ovale, A. auricularium, A. maculatum e Ixodes affinis (Rodríguez-Vivas et al., 2016). Se cree que las garrapatas $R$. sanguineus s.l. (Tinoco-Gracia et al., 2008) e I. affinis son las responsables de la transmisión de B. burgdorferi s.l. a los perros. Solís-Hernández et al. (2015) mencionan que la garrapata I. affinis parasita con mayor frecuencia en perros de áreas rurales que tienen acceso a las zonas forestales cuando son llevados de cacería, y por ende se asume que tienen mayor riesgo de infectarse con la espiroqueta que los animales de zonas urbanas.

El acceso de perros a la áreas forestales, las modificaciones de los hábitat silvestres y el cambio climático han afectado la distribución de los reservorios y sus garrapatas y han favorecido la expansión de B. burgdorferi s.l. (Ogden et al., 2006; Solís-Hernández et al., 2015). La detección de Borrelia en perros podría ser un criterio importante para la evaluación del riesgo de la EL en humanos, ya que el perro puede emplearse como indicador epidemiológico para la identificación de nuevos focos de esta enfermedad (Bhide, Travnicek, Curlik, \& Stefancikova, 2004; Little, Heise, Blagburn, Callister, \& MeadLyme, 2010).

En México, la distribución y presencia de genoespecies patógenas de $B$. burgdorferi en perros y sus garrapatas no ha sido investigada, especialmente en áreas rurales de Yucatán donde los perros tienen acceso a las áreas selváticas, por tal motivo, el objetivo del presente estudio fue detectar la presencia y estimar la prevalencia de B. burgdorferi s.l. en perros y sus garrapatas, en comunidades rurales de Yucatán, México, mediante la detección de los genes flaB, p66 and $o s p C$.

\section{MATERIALES Y MÉTODOS}

Área de estudio: El estudio se realizó en las comunidades rurales de Opichen y Tixmehuac en el estado de Yucatán, México. 
Tixmehuac ocupa una superficie de $251.6 \mathrm{~km}^{2}$, con un clima cálido sub-húmedo con lluvias en verano. La temperatura media anual es de $26^{\circ} \mathrm{C}$ y la precipitación pluvial anual es de $1050 \mathrm{~mm}$; la vegetación prevaleciente en la periferia de la comunidad es selva mediana sub-caducifolia. Las principales actividades económicas de 4746 habitantes de la comunidad son la agricultura, la caza y la construcción en diferentes ciudades del estado de Yucatán (INEGI, 2014). Opichen ocupa un área de $268.2 \mathrm{~km}^{2}$ con un clima cálido sub-húmedo con lluvias en verano. La temperatura media anual es de $28^{\circ} \mathrm{C}$ con una precipitación pluvial anual de $1100 \mathrm{~mm}$; la comunidad está rodeada de parches de la selva baja caducifolia, áreas de cultivo "milpas" y pastizales. La comunidad tiene una población de 6285 habitantes, y la principal fuente de ingresos de la familia es la agricultura y la caza, así como la construcción en diferentes ciudades del estado de Yucatán, complementado con actividades como la cría de animales de traspatio (INEGI, 2014).

Selección de viviendas y perros: En cada comunidad se seleccionaron por conveniencia 50 viviendas que tuvieran al menos un perro. Las comunidades se dividieron en cuatro cuadrantes y se tomó como referencia las calles principales que cruzan los poblados de norte a sur y de este a oeste. Cada vivienda seleccionada fue georeferenciada y se solicitó la anuencia de los dueños para participar en el estudio. Cuando un dueño no aceptaba la participación, se seleccionaba otra vivienda. Los muestreos se realizaron de julio a diciembre de 2013, meses de mayor abundancia de garrapatas en perros de la región (Rodríguez-Vivas et al., 2016).

Manejo y toma de muestras en perros: De cada perro (144) se obtuvo una muestra de sangre. Para ello se realizó la antisepsia del área con alcohol isopropílico y se extrajeron 3 $\mathrm{mL}$ de sangre por punción de la vena safena. Las muestras fueron tomadas bajo el mínimo estrés y se siguieron las recomendaciones establecidas en la normatividad vigente para el manejo y la toma de muestras de animales en
México (NOM-062-ZOO-1999) y en presencia del dueño de cada animal. La sangre se colocó en tubos Vacutainer ${ }^{\circledR}$ con Citrato de Sodio. Las muestras se transportaron en neveras con refrigerantes (aproximadamente $4{ }^{\circ} \mathrm{C}$ ) al Campus de Ciencias Biológicas y Agropecuarias de la Universidad Autónoma de Yucatán (CCBAUADY) en un período no mayor a 24 horas para su posterior congelación a $-80{ }^{\circ} \mathrm{C}$.

Recolecta de garrapatas: Todos los perros de las viviendas seleccionadas fueron inspeccionados durante un período de 10 a 15 min con el propósito de recolectar todas las garrapatas encontradas en el animal (una recolecta). Las garrapatas fueron recolectadas bajo el mínimo estrés para los perros y en presencia del dueño de cada animal, y se siguieron las recomendaciones establecidas en la normatividad vigente para el manejo y toma de muestras de animales en México (NOM062-ZOO-1999). La recolecta se realizó por el método de extracción manual mediante el uso de pinzas de punta fina para sujetar y retirar las garrapatas cerca de la piel del perro, sin comprometer el aparato bucal de la garrapata (Gammon \& Salam, 2002). Todas las garrapatas recolectadas de cada perro se colocaron en viales de $50 \mathrm{~mL}$ que contenían una solución de etanol al $70 \%$. Se recolectaron en total 846 garrapatas que fueron transportadas al Laboratorio de Parasitología en el Campus de Ciencias Biológicas y Agropecuarias de la Universidad Autónoma de Yucatán (CCBA-UADY) para su clasificación taxonómica con la ayuda de las claves taxonómicas descritas por Keirans y Litwat (1989) y Guerrero (1996), y por comparación morfológica con ilustraciones disponibles.

Extracción de ADN: Se extrajo el ADN de todas las muestras de sangre de perro y garrapatas recolectadas mediante el uso del kit comercial DNeasy blood and tissue (Qiagen, Valencia, USA) de acuerdo a las instrucciones del fabricante. Posteriormente, las muestras fueron almacenadas a $-80{ }^{\circ} \mathrm{C}$ hasta su procesamiento. 
Diagnóstico molecular: Para el diagnóstico molecular de B. burgdorferi s.l. se utilizó la prueba de reacción en cadena de la polimerasa (PCR por sus siglas en inglés) convencional y anidada. Para todas las reacciones de PCR se utilizó un termociclador (Minicycler, BIO RAD). Para detectar la presencia del género Borrelia en las muestras de sangre y garrapatas, se utilizó como marcador genético la flagelina cromosómica de 41-kDA (flaB) en un PCR convencional, de acuerdo a la metodología descrita por Jaulhac et al. (2000), mediante el uso de los cebadores: F5'AACACACCAGCATCACTTTCAGG3', R5'GAGAATTAACTCCGCCTTGAGAA GG3'. Los productos positivos a flaB, fueron amplificados para identificar B. burgdorferi s.l. mediante el uso de los genes p66 (66kDA) y $\operatorname{osp} C$ (23-kDA) en PCRs anidados, de acuerdo a la metodología descrita por Bunikis et al. (2004a, b).

Para la amplificación del gen p66 se utilizaron los cebadores:

F5'GATTTTTCTATATTTGGACACAT3', R5'TGTAAATCTTATTAGTTTTT CAAG3', Fn5'CAAAAAAGAAACACCCTCAGATCC3', Rn5'CCTGTTTTTAAATAAATTTTTGTAGCATC3'

y para $o s p C$ :

F5'ATGAAAAAGAATACATTAAGTGC3', R5'ATTAATCTTATAATATTGATTTTAATTAAGG3', Fn5'TATTAATGACTTTATTTTTATTTATATCT3', Rn5'TTGATTTTAATTAAGGTTTT TTTGG3'.

El tamaño de los productos esperados para cada uno de los genes flaB, p66 y ospC son $234 \mathrm{pb}, 684 \mathrm{pb}$ y $585 \mathrm{pb}$, respectivamente. Como controles positivos se usó el ADN genómico de B. burgdorferi sensu stricto B31 (cepas MSK5 y A3), B. afzelii (cepa ATCC51567), B. garinii (cepa ATCC51383) y B. turicatae (cepas TCBP2 y 91E135), proporcionados por la Universidad de Texas A\&M en College Station, Texas (EUA), y para evitar posible contaminación, en cada reacción se incluyó un control negativo. Para cada producto de PCR se realizó electroforesis, a través de un gel de agarosa al $2 \%$, el cual se tiñó con bromuro de etidio, y posteriormente, se examinó mediante transiluminación UV.

Se obtuvo la prevalencia de perros y garrapatas positivos a $B$. burgdorferi s.l. mediante el uso de los marcadores genéticos flaB, p66 y $o s p C$. Para conocer la diferencia en la frecuencia de perros y garrapatas positivas a $B$. burgdorferi s.l. en ambas comunidades (Opichen y Tixmehuac) se usó la prueba de $\mathrm{X}^{2}$.

\section{RESULTADOS}

Se muestrearon un total de 144 perros, 88 en la comunidad de Opichen y 56 en la comunidad de Tixmehuac. De estos, se recolectaron un total de 846 garrapatas, 672 en la comunidad de Opichen y 174 en la comunidad de Tixmehuac. De los 144 perros estudiados, en 139 perros se recolectaron garrapatas de una o más especies (1-12 garrapatas por animal). La cabeza, orejas y extremidades fueron las zonas de perros donde se recolectaron el mayor número de garrapatas. Treinta y tres garrapatas $I$. affinis fueron recolectadas en 16 perros, 27 del género Amblyomma de 22 perros (A. maculatum, A. ovale, A. auricularium y $A$. mixtum) y 786 garrapatas $R$. sanguineus s.l. de 91 perros (Cuadro 1).

Análisis molecular: Los resultados del análisis molecular en sangre de perro, muestran que el 25 / 144 (17.3\%) fueron positivos al gen flaB, de los cuales 13 / 88 (14.7\%) corresponden a la comunidad de Opichen y 12 / 56 (21.4 $\%$ ) a la comunidad de Tixmehuac (Cuadro 2). No se encontró diferencia significativa ( $p>$ $0.05)$ entre las dos comunidades. Del total de muestras, $12.50 \%(18 / 144)$ resultaron positivas al gen $p 66$ y $1.38 \%(2 / 144)$ al gen $\operatorname{ssp} C$.

De las 846 garrapatas recolectadas, $1.53 \%$ (13 / 846) fueron positivas al gen flaB (Cuadro 2). En la comunidad de Tixmehuac la prevalencia fue mayor que en la comunidad de Opichen $(\mathrm{p}<0.05)$. De las especies de garrapatas analizadas $R$. sanguineus s.l. tuvo una prevalencia de infección de $0.89 \%$ (7 / 786), A. mixtum de $5.88 \%(1 / 17)$ e $I$. affinis de $15.15 \%$ (5/33) 
CUADRO 1

Abundancia de garrapatas recolectadas por especie, en perros de dos comunidades rurales de Yucatán, México

TABLE 1

Abundance of tick species collected in dogs of two rural communities in Yucatán, Mexico

\begin{tabular}{|c|c|c|c|c|}
\hline Género & Especie & Tixmehuac & Opichen & Total \\
\hline Rhipicephalus & sanguineus s.l. & 146 & 640 & 786 \\
\hline Amblyomma & maculatum & 1 & 5 & 6 \\
\hline Amblyomma & ovale & 1 & 1 & 2 \\
\hline Amblyomma & auricularium & 1 & 1 & 2 \\
\hline Amblyomma & mixtum & 6 & 11 & 17 \\
\hline Ixodes & affinis & 19 & 14 & 33 \\
\hline Total & & 174 & 672 & 846 \\
\hline
\end{tabular}

\section{CUADRO 2}

Resultados correspondientes al PCR del gen $f l a B$, en muestras de sangre de perros y garrapatas de las localidades rurales de Opichen y Tixmehuac en Yucatán, México

TABLE 2

PCR results for the $f l a B$ gene in blood samples and ticks of dogs from the rural communities of Opichen and Tixmehuac in Yucatán, Mexico

\begin{tabular}{|c|c|c|c|c|c|c|}
\hline Poblado & $\begin{array}{c}\text { Perros } \\
\text { muestreados }\end{array}$ & $\begin{array}{c}\text { Perros } \\
\text { positivos a } f l a B\end{array}$ & $\begin{array}{c}\text { Prev. } \\
\text { B. burgdorferi s.l. }\end{array}$ & $\begin{array}{l}\text { Garrapatas } \\
\text { recolectadas }\end{array}$ & $\begin{array}{c}\text { Garrapatas } \\
\text { positivas flaB }\end{array}$ & $\begin{array}{c}\text { Prev. } \\
\text { B. burgdorferi s.l. }\end{array}$ \\
\hline Opichen & 88 & 13 & $14.7 \%$ & 672 & 5 & $1 \%$ \\
\hline Tixmehuac & 56 & 12 & $21.4 \%$ & 174 & 8 & $4.6 \%$ \\
\hline Total & 144 & 25 & $17.3 \%$ & 846 & 13 & $1.53 \%$ \\
\hline
\end{tabular}

Prev: Prevalencia, s.l.: sensu lato.

Prev: Prevalence, s.l.: sensu lato.

(Cuadro 3), siendo esta última especie la que presentó mayor prevalencia $(\mathrm{p}<0.05)$. Solamente una garrapata $R$. sanguineus s.l. fue positiva al gen $p 66$ y ninguna especie de garrapata fue positiva al gen $o s p C$.

\section{DISCUSIÓN}

El perro puede actuar como un importante centinela del complejo B. burgdorferi s.l., debido al contacto directo que mantiene con el hábitat de las garrapatas vectoras, haciéndolo más vulnerable a adquirir esta bacteria (Goossens, van den Bogaard, \& Nohlmans, 2001). Asimismo, los perros desempeñan un papel clave en la dispersión de garrapatas, transportándolas al ambiente intra o peridomiciliar (Appel, 1990; Straubinger 2000; De
Lacerda, Cuhna, Antunes, Do, \& Machado, 2005), lo cual ocasiona un riesgo para la población humana, ya que incrementa su exposición con el agente (Mather, Fish, \& Coughlin, 1994). Los habitantes de ambas comunidades rurales que trabajan en el campo y tienen acceso a áreas selváticas o agrícolas, podrían tener mayor riesgo de adquirir el agente, ya que pueden tener mayor exposición a garrapatas infectadas con las espiroquetas (Stanek, Wormser, Gray, \& Strle, 2012).

En México, la mayoría de los estudios realizados sobre el diagnóstico de $B$. burgdorferi s.l. en perros, se han basado principalmente en pruebas serológicas. Salinas-Meléndez et al. (1999) reportaron una seroprevalencia del 16 $\%$ en perros de Monterrey, y Tinoco-Gracia et al. $(2007 ; 2008)$ del $8.2 \%$ y $6.8 \%$ en Mexicali. 
CUADRO 3

Perros y sus garrapatas positivas al gen flaB obtenidas dos localidades rurales de Yucatán, México

TABLE 3

Dogs and their positive ticks to flaB gene obtained from two communities in Yucatán, Mexico

\begin{tabular}{|c|c|c|c|c|}
\hline \multicolumn{3}{|c|}{ Perro } & \multicolumn{2}{|l|}{ Garrapatas } \\
\hline Identificación & flaB & p66 & Especie & flaB \\
\hline 1 & + & + & Ixodes affinis & - \\
\hline 2 & + & + & Rhipicephalus sanguineus s.l. & - \\
\hline 3 & + & + & Ixodes affinis & + \\
\hline 4 & + & + & $*$ & $*$ \\
\hline 5 & + & + & $*$ & $*$ \\
\hline 6 & + & + & $*$ & * \\
\hline 7 & + & + & Rhipicephalus sanguineus s.l. & - \\
\hline 8 & + & + & $*$ & * \\
\hline 9 & + & + & Rhipicephalus sanguineus s.l. & - \\
\hline 10 & + & - & Rhipicephalus sanguineus s.l. & - \\
\hline 11 & + & - & Rhipicephalus sanguineus s.l. & - \\
\hline 12 & + & + & Rhipicephalus sanguineus s.l. & - \\
\hline 13 & + & + & Rhipicephalus sanguineus s.l. & - \\
\hline 14 & + & + & $*$ & * \\
\hline 15 & + & + & Amblyomma mixtum & - \\
\hline 16 & + & - & $*$ & * \\
\hline 17 & + & + & Ixodes affinis & + \\
\hline 18 & + & + & Rhipicephalus sanguineus s.l. & - \\
\hline 19 & + & - & Rhipicephalus sanguineus s.l. & - \\
\hline 20 & + & + & Rhipicephalus sanguineus s.l. & - \\
\hline 21 & + & - & Rhipicephalus sanguineus s.l. & - \\
\hline 22 & + & + & $*$ & * \\
\hline 23 & + & - & Amblyomma mixtum & - \\
\hline 24 & + & + & Rhipicephalus sanguineus s.l. & - \\
\hline 25 & + & - & Ixodes affinis & - \\
\hline
\end{tabular}

*No se encontraron garrapatas en los perros. Las garrapatas A. mixtum y R. sanguineus s.l. positivas al gen flaB fueron recolectadas de perros negativos. Tres $I$. affinis positivos a flaB fueron recolectados de perros negativos.

* No ticks were found in dogs. The ticks $A$. mixtum and $R$. sanguineus s.l. positive to the flaB gene were collected from negative dogs. Three $I$. affinis positive to fla $\mathrm{B}$ were collected from negative dogs.

Actualmente, la borreliosis canina en México es poco estudiada, debido a que los perros infectados con Borrelia spp. generalmente no desarrollan signos clínicos asociados con esta espiroqueta, los que hace más difícil su detección, monitoreo y tratamiento (Little et al., 2010). Sin embargo, en algunas ocasiones los perros infectados con la bacteria pueden presentar artritis migratoria, claudicación intermitente, anorexia y malestar general. Asimismo se ha reportado en perros bloqueo cardíaco, signos neurológicos, convulsiones y falla renal fatal (Bhide et al., 2004).
Los principales vectores de B. burgdorferi s.l. son las garrapatas del género Ixodes. En EE.UU. es transmitida principalmente por $I$. scapularis, I. pacificus e I. neotomae, mientras que la mayor parte de Europa (centro, norte y oeste) es transmitido por I. ricinus, y en Europa del este y Asia por I. persulcatus (Stanek et al., 2012). Recientemente, en EE.UU. se ha reportado que $I$. affinis puede ser vector de $B$. burgdorferi s.l. (Harrison et al., 2010). En el presente estudio, se encontró que el $15.15 \%$ de $I$. affinis estudiados fueron positivos al gen flaB (dos perros y sus garrapatas I. affinis 
fueron positivos) lo que pone de manifiesto el probable papel de esta especie de garrapata en el ciclo de transmisión de esta bacteria a los perros. La prevalencia de infección de garrapata es variable dependiendo de la especie, las fases de desarrollo y las condiciones medioambientales. En un estudio realizado en Servia, Savić et al. (2010) encontraron que el $22.1 \%$ de garrapatas I. ricinus recolectadas en perros, se encontraban infectadas con $B$. burgdorferi s.l. En otro estudio realizado en EE.UU. el porcentaje de infección de I. scapularis con Borrelia spp. alcanzó hasta $50 \%$ en garrapatas adultas (Greene \& Straubinger, 2006). Generalmente, el porcentaje de infección de las garrapatas ixódidas se incrementa con el ciclo de vida de los vectores ( $\sim 10 \%$ en ninfas, $\sim 20$ $\%$ en adultas) y puede alcanzar hasta $75 \%$ en el centro de Europa (Rauter \& Hartung, 2005).

Se sabe que las fases inmaduras de $I$. affinis parasitan principalmente a pequeños mamíferos y aves, mientras que la fase adulta parasita a mamíferos de talla mediana y grande (Guglielmone, Estrada-Peña, Keirans, \& Robbins, 2004; Mannelli et al., 2012). I. affinis generalmente no infesta humanos (Rudenko et al., 2012); sin embargo, Allan (2001) observó que esta especie puede ocasionalmente alimentarse de humanos. En futuros estudios se recomienda investigar el papel de $I$. affinis como vector competente de B. burgdorferi s.l. en animales y humanos en comunidades rurales de Yucatán, México.

Aunque con menor frecuencia, en el presente estudio se encontraron $R$. sanguineus s.l. y A. mixtum positivos al gen flaB de B. burgdorferi s.l., ambas especies de garrapatas no son reconocidas ampliamente como vectores competentes de EL; sin embargo, Schulze et al. (1984), mencionan que las garrapatas de género Amblyomma pueden considerarse como vector potencial de Borrelia en América del Sur y México. En futuros estudios, será necesario confirmar el papel de estas especies de garrapatas como vectores competentes de la EL en Yucatán, México, ya que $R$. sanguineus s.l. y $A$. mixtum son las garrapatas más abundantes que parasitan a una amplia gama de mamíferos incluyendo al humano en Yucatán (RodríguezVivas et al., 2016). Asimismo, estas dos especies de garrapatas pueden ser vectores de varios agentes zoonóticos tales como Coxiella burnetii, Ehrlichia canis, R. conorii, and Rickettsia rickettsii, y pueden representar un riesgo de transmisón a la población de las comunidades estudiadas (Dantas-Torres, 2008; RodríguezVivas et al., 2016).

La presencia de perros positivos a B. burgdorferi s.l. en perros y sus garrapatas en comunidades rurales de Yucatán, México, ponen de manifiesto la importancia de este agente en el sureste de México y el posible riesgo de transmisión a los humanos. En el 2007 se reportaron los primeros casos de EL autóctonos en humanos de México, que presentaron manifestaciones cutáneas y neurológicas procedentes del Valle de México y Quintana Roo (Gordillo-Pérez et al., 2007).

Este estudio confirma la existencia de $B$. burgdorferi s.l. en perros y sus garrapatas en comunidades rurales de Yucatán, México. La potencial transmisión de B. burgdorferi s.l. a los humanos es alta, debido a que en Yucatán existen las condiciones ambientales y ecológicas para la proliferación de garrapatas y es reconocido que en área rurales existe una alta infestación de humanos con garrapatas. Se necesitan realizar estudios futuros para conocer la presencia de la EL en la población humana de comunidades rurales de Yucatán, México.

\section{AGRADECIMIENTOS}

Los autores agradecen a los habitantes y autoridades de las comunidades de Opichén y Tixméhuac por permitirnos entrar en sus casas y por su ayuda durante el Proyecto. Nuestra gratitud a Alonso Panti May, Rodrigo Carrillo Peraza y Marco Torres Castro, por su apoyo técnico.

\section{RESUMEN}

Actualmente, el estudio de la borreliosis canina adquiere mayor relevancia, ya que el perro es considerado como un importante centinela del complejo Borrelia 
burgdorferi sensu lato, el cual podría desempeñar un papel clave en la dispersión de garrapatas de las áreas selváticas al ambiente doméstico. En México, la distribución y presencia de genoespecies patógenas de B. burgdorferi en perros y sus garrapatas aún no ha sido investigada. Por tal motivo, la presente investigación tiene como objetivo detectar y estimar la prevalencia de B burgdorferi s.l. en perros y sus garrapatas en dos comunidades rurales de Yucatán, México. En cada comunidad se visitaron 50 viviendas donde se estudiaron 144 muestras de sangre de perros por punción de la vena safena, así como la colecta de sus garrapatas. Se colectaron un total de 846 garrapatas de las especies Ixodes affinis (33 / 846), Rhipicephalus sanguineus sensu lato (786 / 846) y Amblyomma mixtum (27 / 846). Para detectar la presencia de B. burgdorferi s.l. en dichas muestras, se amplificó el gen conservado $f l a B$ y las lipoproteínas de membrana externa, osp $C$ y $p 66$, mediante el uso de la reacción en cadena de la polimerasa. La prevalencia obtenida en sangre de perros fue de $17.3 \%$ (25/144) para flaB, $12.50 \%(18 / 144)$ para el gen $p 66 \mathrm{y}$ $1.38 \%$ (2 / 144) para el gen ospC. De las garrapatas analizadas, $R$. sanguineus s.l. tuvo una prevalencia de infección de $0.89 \%$, A. mixtum de $5.88 \%$ e I. affinis de $15.15 \%$, siendo esta última especie la que presentó mayor prevalencia. Dos perros y sus garrapatas I. affinis fueron positivos al gen flaB. Solamente una garrapata $R$. sanguineus s.l. fue positiva al gen $p 66$ y ninguna especie de garrapata fue positiva al gen $\operatorname{sp} C$. Este estudio confirma la existencia de $B$. burgdorferi s.l. en perros y sus garrapatas en comunidades rurales de Yucatán, México. La detección de Borrelia en perros podría ser un criterio importante para la evaluación del riesgo de borreliosis en humanos, ya que el perro puede emplearse como indicador epidemiológico para la identificación de nuevos focos de esta enfermedad.

Palabras clave: Borrelia burgdorferi s.l., Enfermedad de Lyme, perros, Ixodes affinis, Rhipicephalus sanguineus s.l., Amblyomma mixtum.

\section{REFERENCIAS}

Allan, S. (2001). Ticks (Class Arachnida: Order Acarina). In W. S. Samuel, M. J., Pybus, \& A. A. Kocan (Eds.), Parasitic Diseases of Wild Animals. 2nd edition (pp. 72-106), Iowa: Iowa State University Press.

Appel, J. G. (1990). Lyme disease in dogs and cats. Compendium, 12, 617.

Arroyave, C. M., \& Támez, G. R. (1994). Enfermedad de Lyme. Informe de dos casos. Boletín Médico del Hospital Infantil de México, 51, 117-120.

Bhide, M., Travnicek. M., Curlik. J., \& Stefancikova, A. (2004). The importance of dogs in eco-epidemiology of Lyme borreliosis: a review. Veterinary Medicine, 4, 135-142.
Bunikis, J., Garpmo, U., Tsao, J., Berglund, J., Fish, D., \& Barbour, A. G. (2004a). Sequence typing reveals extensive strain diversity of the Lyme borreliosis agents Borrelia burgdorferi in North America and Borrelia afzelii in Europe. Microbiology, 150, 1741-1755.

Bunikis, J., Tsao J., Luke C. J., Luna, M. G., Fish, D., \& Barbour, A. G. (2004b). Borrelia burgdorferi infection in a natural population of Peromyscus leucopus mice: a longitudinal study in an area where Lyme Borreliosis is highly endemic. Journal of Infectious Diseases, 189, 1515-1523.

Burgdorfer, W., Hayes, S. F., \& Corwin, D. (1989). Pathophysiology of the Lyme disease spirochete, Borrelia burgdorferi, in ixodid ticks. Clinical Infectious Diseases, 6, S1442-S1450.

Centers for Disease Control and Prevention (CDC). (2015). Lyme Disease. Retrieved from ww.cdc.gov/lyme

Dantas-Torres, F. (2008). The brown dog tick, Rhipicephalus sanguineus (Latreille, 1806) (Acari: Ixodidae): from taxonomy to control. Veterinary Parasitology, $152,173-185$.

De Lacerda, A. A., Cuhna, M. R., Antunes, D. R., Do, N. C. F., \& Machado, B. R. (2005). Frequency of Ixodes ricinus ticks in Europe: a metaanalysis. Applied and Environmental Microbiology, 71, 7203-7216.

Feria, T. P., Castro, I., Gordillo, G., Cavazos, A. L., Vargas, M., Grover, A., ... Esteve-Gassent, M. D. (2014). Implications of climate change on the distribution of the tick vector Ixodes scapularis and risk for Lyme disease in the Texas-Mexico transboundary region. Parasites \& Vectors, 7, 199.

Gammon, M., \& Salam, G. (2002). Tick removal. American Family Physician, 66, 643-645.

Glenny, M., Mendoza, L., \& Falconi, E. (2004). Detección de anticuerpos contra Borrelia burgdorferi e identificación de garrapatas ixodidas en Piura y Amazonas, Perú. Revista Peruana de Medicina Experimental y Salud Pública, 20, 23-27.

Goossens, H. A. T., van den Bogaard, A. E., \& Nohlmans, M. K. E. (2001). Dogs as sentinel for human Lyme borreliosis in The Netherlands. Journal of Clinical Microbiology, 39, 844-848.

Gordillo-Pérez, G., Torres, J., Solórzano-Santos, F., De Martino, S., Lipsker, D., Velázquez, E., Ramon, G., Muñoz, O., \& Jaulhac, B. (2007). Borrelia burgdorferi infection and cutaneous Lyme disease, Mexico. Emerging Infectious Diseases, 13, 1556-1558.

Greene, C. E., \& Straubinger, R. K. (2006). Borreliosis. In C. E. Greene (Ed.), Infectious Diseases of the Dog and Cat. 3rd edition (pp. 417-435). Philadelphia: WB Saunders Elsevier. 
Guerrero, R. (1996). Las garrapatas de Venezuela (Acarina: Ixodidae). Listado de especies y claves para su Identificación. 1996. Boletín de Malariología y Salud Ambiental, 36, 1-24.

Guglielmone, A., Estrada-Peña A., Keirans, J., \& Robbins, R. (2004). Las garrapatas (Acari: Ixodida) de la región zoogeográfica neotropical. Argentina: Instituto Nacional de Tecnología Agropecuaria.

Harrison, B. A., Rayburn, W. H. Jr, Toliver, M., Powell, E. E., Engber, B. R., Durden, L. A., ... Whitt, P. B. (2010). Recent discovery of widespread Ixodes affinis (Acari: Ixodidae) distribution in North Carolina with implications for Lyme disease studies. Journal of Vector Ecology, 35, 174-179.

Instituto Nacional de Estadística y Geografía (INEGI). (2014). Censo General de Población y Vivienda 2010. Recuperado de www.inegi.org.mx

Jaulhac, B., Heller, R., Limbach, F. X., Hansmann, Y., Lipsker, D., Monteil, H., ... Piémont, Y. (2000). Direct molecular typing of Borrelia burgdorferi sensu lato species in synovial samples from patients with Lyme arthritis. Journal of Clinical Microbiology, 38,1895 .

Keirans, J. E., \& Litwak, T. R. (1989). Pictorial key to the adults of hard ticks, family Ixodidae (Ixodida: Ixodidea), east of the Mississippi river. Journal Medical of Entomology, 26, 435-448.

Krupka, I., \& Straubinger, R. K. (2010). Lyme borreliosis in dogs and cats: background, diagnosis, treatment and prevention of infections with Borrelia burgdorferi sensu stricto. Veterinary Clinics of North America: Small Animal Practice, 40, 1103-1119.

Levy, S. A., \& Magnarelli, L. A. (1992). Relationship between development of antibodies to Borrelia burgdorferi in dogs and the subsequent development of limb/ joint borreliosis. Journal of the American Veterinary Medical Association, 200, 344-347.

Little, S. E., Heise, S. R., Blagburn, B. L., Callister, S. M., \& MeadLyme, P. S. (2010). Borreliosis in dogs and humans in the USA. Trends in Parasitology, $26,213-218$.

Mannelli, A., Bertolotti, L., Gern, L., \& Gray, J. (2012). Ecology of Borrelia burgdorferi sensu lato in Europe: transmission dynamics in multi-host systems, influence of molecular processes and effects of climate change. Microbiological Reviews, 36, 837-61.

Mather, T. N., Fish, D., \& Coughlin, R. T. (1994). Competence of dogs as reservoir for Lyme disease spirochetes (Borrelia burgdorferi). Journal of the American Veterinary Medical Association, 205, 186-188.

Ogden, N. H., Maarouf, A., Barker, I. K., Bigras-Poulin, M., Lindsay, L. R., Morshed, M. G., O'callaghan, C. J., ... Charron, D. F. (2006). Climate change and the potential for range expansion of the Lyme disease vector Ixodes scapularis in Canada. International Journal for Parasitology, 36, 63-70.

Rauter, C., \& Hartung, T. (2005). Prevalence of Borrelia burgdorferi sensu lato genospecies in Ixodes ricinus Ticks in Europe: a Metaanalysis. Applied and Environmental Microbiology, 71, 203-7216.

Rodríguez-Vivas, R. I., Apanaskevich, D. A., Ojeda-Chi, M. M., Trinidad-Martínez, I., Reyes-Novelo, E., Esteve-Gassent, M. D., \& Pérez de León, A. A. (2016). Ticks collected from humans, domestic animals, and wildlife in Yucatan, Mexico. Veterinary Parasitology, 215, 103-107.

Rudenko, N., Golovchenko, M., Hönig, V., Mallátová, N., Krbková, L., Mikulášek, P., ... Oliver, J. H. Jr. (2012). Detection of Borrelia burgdorferi sensu stricto OspC alleles associated with human Lyme Borreliosis worldwide in non-human-biting tick Ixodes affinis and rodent hosts in Southeastern United States. Applied and Environmental Microbiology, 79, 1444-1453.

Salinas-Meléndez, J. A., Ávalos-Ramírez, R., RiojasValdez, V. M., \& Martínez-Muñoz, A. (1999). Serological survey of canine borreliosis. Revista Latinoamericana de Microbiología, 41, 1-3.

Salinas-Meléndez, J. A., Tamez-González, R., Welsh-Lozano, O., \& Barrera-Saldaãna, H. A. (1995). Detection of Borrelia burgdorferi DNA in human skin biopsies and dog sinovial fluid by the polymerase chain reaction. Revista Latinoamericana de Microbiologia, 37, 7-10.

Savić, S., Vidić, B., Lazić, S., Lako, B., Potkonjak, A., \& Lepšanović, Z. (2010). Borrelia burgdorferi in ticks and dogs in the province of Vojvodina, Serbia. Parasite, 17, 357-361.

Schulze, T. L., Bowen, G. S., Bosler, E. M., Lakat, M. F., Parkin, W. E., Altman, R., ... Shisler, J. K. (1984). Amblyomma americanum: a potential vector of Lyme disease in New Jersey. Science, 224, 601-603.

Smith, R. P. Jr, Rand, P. W., Lacombe, E. H., Telford, S. R, Rich, S. M., Piesman, J., \& Spielman, A. (1993). Norway rats as reservoir hosts for Lyme disease spirochetes on Monhegan Island, Maine. Journal Infected Disease, 168, 687-691.

Solís-Hernández, A., Rodríguez-Vivas, R. I., BarreraPérez, M. A., Esteve-Gassent, M. D., \& Apanaskevich, D. A. (2015). Ixodes affinis (Acari: Ixodidae) in dogs from rural localities of Yucatán, México: Prevalence, abundance and associated factors. Veterinaria México, 2, 1-9.

Stanek, G., Wormser, G. P., Gray, J., \& Strle, F. (2012). Lyme borreliosis. The Lancet, 379, 461-473. 
Straubinger, R. K. (2000). PCR-based quantification of Borrelia burgdorferi organisms in canine tissues over a 500-day postinfection period. Journal of Clinical Microbiology, 38, 2191-2199.

Tinoco-Gracia, L., Quiroz-Romero, H., Quintero, M. T., Renteria-Evangelista, T. B., Barreras-Serrano, A., Hori-Oshima, S., ... Vinasco, J. (2007). Seroprevalence of Borrelia burgdorferi in dogs from a
Mexico-U.S. border desert region: pilot study. Journal of Animal and Veterinary Advances, 6, 787-789.

Tinoco-Gracia, L., Quiroz-Romero, H., Quintero, M. T., Rentería-Evangelista, T. B., Barreras-Serrano, A., Hori-Oshima, S., ... Moro, M. H. (2008). Prevalence and risk factors for Borrelia burgdorferi infection in Mexicali, Baja California, a Mexico-US border city. International Journal of Applied Research in Veterinary Medicine, 6, 161-165. 